\title{
5
}

\section{The Composite Species Concept: A Rigorous Basis for Cladistic Practice}

\author{
D. J. Kornet and James W. McAllister
}

\begin{abstract}
As previous work has shown, the genealogical network can be partitioned exhaustively into internodons, mutually exclusive and historically continuous entities delimited between two successive permanent splits or between a permanent split and an extinction. Internodons are not suitable candidates for the status of species, because of their short life span and the difficulty of recognizing their boundaries. However, internodons may be suitable building blocks for a viable species concept. We introduce the concept of composite species as a sequence of internodons, by qualifying only some permanent splits in the genealogical network as speciation events. The permanent splits that count as speciation events on our account are those associated with a character state fixation: this proposal ensures the recognizability of composite species. Lastly, we show how actual taxonomic practice is able to recover the phylogenetic tree of composite species from standard morphological data.
\end{abstract}

Keywords: Species concepts, genealogical network, internodons, character state, fixation, speciation.

\subsection{INTRODUCTION}

This paper presents a new species concept, the composite species concept, which is developed from first principles. On this concept, species are historical entities composed of parts of the genealogical network named internodons, and their recognition in nature is achievable by standard taxonomic practice. The composite species concept shows some affinity with the phylogenetic species concept, but has important advantages over it.

The main motivation for developing the composite species concept is the conviction that a good species concept ought to define species that are mutually exclusive and that exhaust the genealogical network. The composite species concept fulfils this requirement. Available species concepts, by contrast, including the phylogenetic species concept, fail to satisfy this condition. Furthermore, the composite species concept incorporates rigorous 
definitions of taxonomic entities and phenomena, including character state and fixation, which make it more precise than available rivals.

We begin in Section 5.2 by reviewing the concept of internodon. This may be characterized informally as a part of the genealogical network delimited by two successive permanent splits, or by a permanent split and an extinction. However, a formal definition of internodon in terms of an equivalence relation is also available. Some authors, most notably Hennig, have proposed that entities approximating to internodons constitute species. In contrast, we argue that internodons are not suitable candidates for the status of species, because of their short life span and the fact that it is difficult to recognize or diagnose them with the aid of standard taxonomic data.

We use the concept of internodon in Section 5.3 to formulate the composite species concept. In informal terms, a composite species is a lineage of internodons descended from an internodon with a particular property, which we call quality $Q$. We opt initially for an abstract approach, in which we explore implications of this definition of composite species before identifying quality $Q$ with an actual property of internodons. We argue that it is desirable that species be paraphyletic groups of lower-level entities, and point out that composite species are paraphyletic groups of internodons.

In Section 5.4, we fill in quality $Q$ in morphological terms: on our candidate definition, an internodon has quality $Q$ if and only if a character state reaches fixation in it. Because the concepts of character state and of fixation are not clearly defined or characterized in the theoretical systematics literature, we provide our own account of these concepts (Section 5.5). We describe various phenomena that can be expected to arise in the fixation of character states and discuss their effect on phylogeny reconstruction. We provide concise definitions of internodon and composite species for easy reference in Section 5.6.

In Section 5.7, we contrast composite species with morphological species, pointing out that - although our preferred candidate for quality $Q$ is defined in morphological terms - possession of a certain morphological attribute is neither a necessary nor a sufficient condition for an organism to belong to a certain composite species. We then show, with the aid of a hypothesized phylogenetic tree of internodons, that the composite species concept is compatible with standard phylogeny reconstruction techniques in cladistics, and that the concept is capable of placing this practice on a more rigorous footing (Section 5.8).

In Section 5.9, we point out that composite species arise not by a symmetrical splitting up which gives rise to two descendant sibling species, but by the asymmetrical process of branching off, in which the ancestor species survives. We argue that this is a further element that weighs in favour of the concept of composite species, as it is undesirable that species be regarded as arising by dichotomous splits. We compare the composite species 
concept with the phylogenetic species concept in Section 5.10: we regard the latter as an imperfect approximation and operationalization of the more rigorous composite species concept. The paper concludes with some remarks on the evolutionary behaviour of composite species in Section 5.11.

\subsection{INTERNODONS: BUILDING BLOCKS FOR COMPOSITE SPECIES}

The genealogical network is the mapping of all actual organisms and the parental relationships holding between them. The problem of how to define species can be interpreted as the question of how to partition the genealogical network into supra-organismal entities that meet most of our pre-analytic intuitions about species. Among these intuitions is the conviction that all organisms belong to precisely one species, or, in other words, that species are mutually exclusive and exhaustive of the genealogical network.

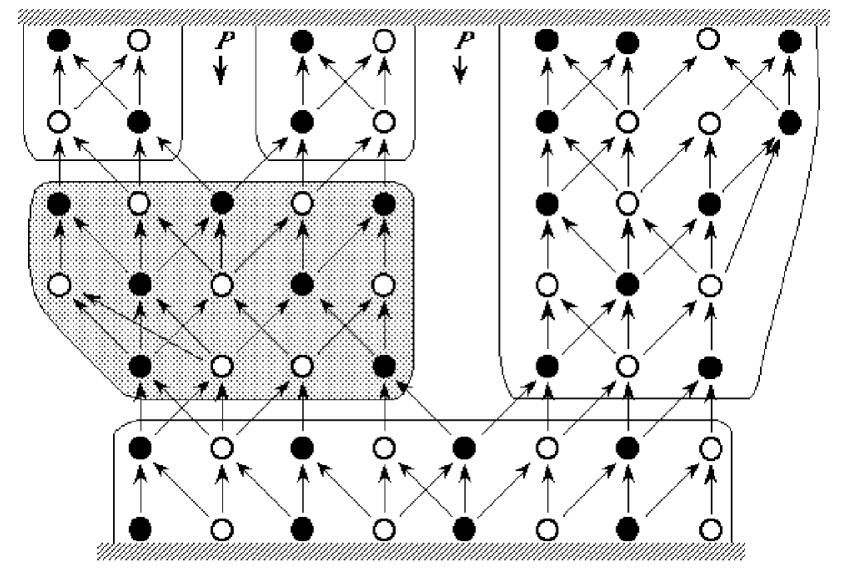

Figure 5.1. A part of the genealogical network with two permanent splits $(\boldsymbol{P})$ marking the beginning and end of the life span of a historical supra-organismal entity (shaded), which we call internodon.

One way of partitioning the genealogical network into parts that are mutually exclusive and exhaustive is by dividing it into portions delimited by two successive permanent splits (Figure 5.1) or by a permanent split and an extinction. These entities will be called internodons in this paper. Kornet (1993) has shown that internodons can be defined formally as sets of organisms between any two of which a particular relation INT holds. The properties of relation INT guarantee that internodons have temporal continuity. Furthermore, INT is, in mathematical terms, an equivalence relation: it is 
reflexive, symmetric and transitive. Since an equivalence relation partitions its domain into exhaustive and mutually exclusive sets, we can be certain that internodons are exhaustive of the genealogical network and mutually exclusive (Kornet et al., 1995). In this way, the concept of internodon meets two important intuitions that we have about species: that species are historically continuous and non-overlapping entities that exhaust the genealogical network.

Does this mean that internodons are suitable candidates for the status of species? This is suggested by the species concept defined by Hennig (1966) and elaborated by later authors. Hennig delimited species by reference to splits in the genealogical network, writing, "New species arise when gaps develop in the fabric of the tokogenetic relationships" (Hennig, 1966: 30). Hennig's species thus resemble internodons, though Hennig added interbreeding ability as a defining criterion for species (ibid.: 45) and stipulated a constant conjunction between splits in the genealogical network and morphological diversification (ibid.: 88). Ridley (1989) elaborates on Hennig's concept of species, coming closer to the notion of internodon by rejecting morphology and interbreeding ability as defining criteria in his "cladistic species concept" (Ridley, 1989: 5 and 11), named "internodal species concept" by Nixon and Wheeler (1990: 213).

Ridley's cladistic species could be made identical to internodons by stipulating that they arise only with permanent splits in the genealogical network. Ridley, by contrast, commits himself to the thesis that temporary splits too are speciation events, stating that hybridization followed by merging is a speciation event (Ridley, 1989: 4-5). A merging of two of his cladistic species, each consisting of a separate branch of the genealogical network, is nothing other than a closing up of a temporary split, in which Ridley must suppose that the two cladistic species which later "merge" originated.

It matters a great deal whether one considers temporary splits in the genealogical network to be speciation events. Temporary splits in the network are very frequent (Figure 5.2): they open up between, for instance, any pair of siblings that do not immediately interbreed. Because of their frequency, it is implausible to suggest that temporary splits constitute speciation events. Nor can we distinguish in a principled way between "short" and "long" temporary splits, with the intention of giving the status of speciation event only to the latter: there is a continuous gradation in the length of temporary splits, as Figure 5.2 illustrates.

Of course, when a split first appears in the network, it is impossible to tell whether it will be permanent or temporary. A permanent split is recognizable only retrospectively: the conclusive criterion for deeming a split permanent is the extinction of one of the branches in which the split has resulted. We depend on retrospective diagnosis to identify with certainty any historical entity in the genealogical network. 
Notwithstanding Hennig's and Ridley's apparent belief that an entity resembling internodons should be seen as species, there are two important reasons for rejecting the concept of internodon as an acceptable concept of species: one is practical and one more fundamental.

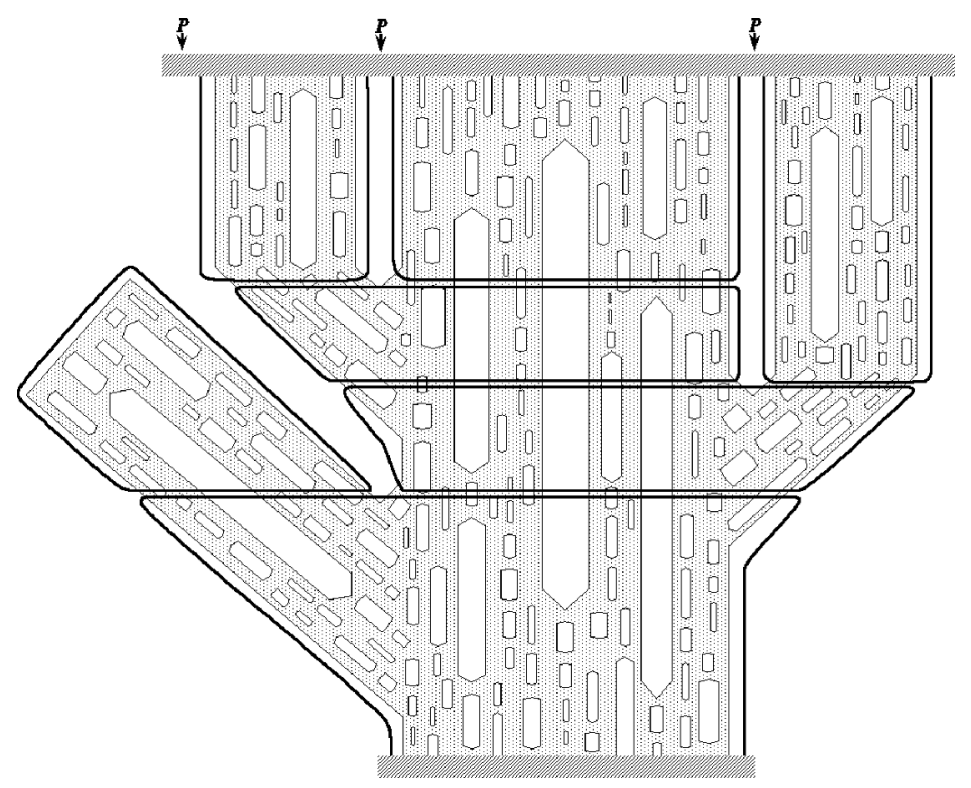

Figure 5.2. A part of the genealogical network with a split made permanent by an extinction, two further splits which we assume to be permanent, and many temporary splits. The life span of internodons is delimited only by permanent splits and extinctions.

The practical objection is the following. Whether a given organism belongs to a given internodon depends solely on the structure of the genealogical network and the organism's position in it. It does not depend on, for instance, the morphological characteristics of the organisms, either macromorphological or genetic. Since our knowledge of the tokogenetic relationships among organisms (which constitute the genealogical network) is typically scarce, and reference to morphological characteristics is in principal irrelevant to determining to which internodon an organism belongs, internodons have low recognizability. The internodal concept would therefore have very limited practical value as a species concept.

The more fundamental objection against interpreting internodons as species lies in their short life span. Both Hennig and Ridley seem to have overestimated the typical life span of their species, assuming that they extend over many generations of organisms. Hennig writes: "Species are relatively 
stable complexes that persist over long periods of time, but they are not absolutely permanent" (Hennig, 1966: 19; see also 30). Ridley reproduces and endorses Hennig's diagram showing species living long enough to accumulate several new character states (Ridley, 1989: 3, Figure 1; see also ibid.: 13, Figure 3).
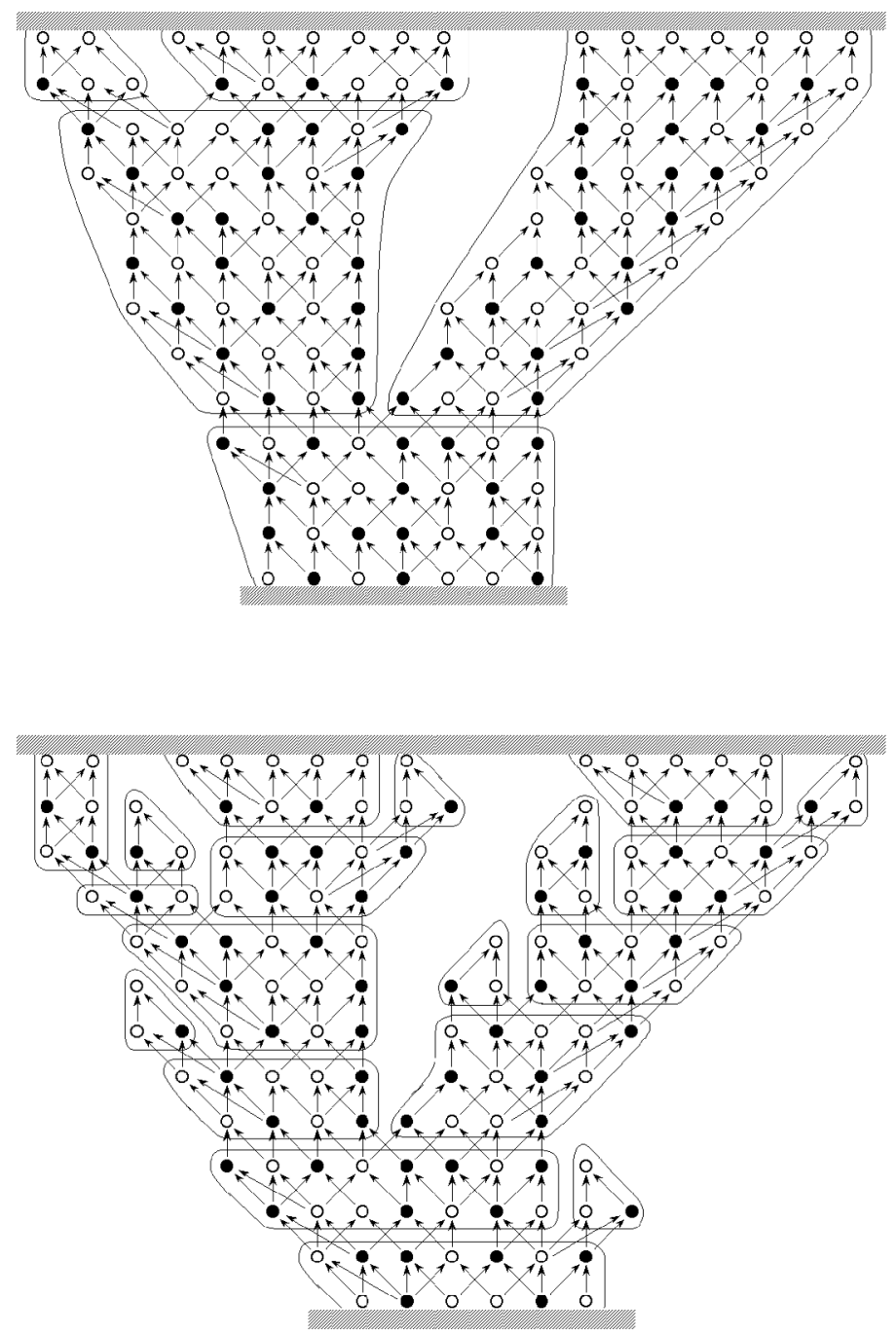

Figure 5.3. The life span of internodons should not be thought of as extended (above), but as relatively short due to the frequent extinction of small groups (below). 
Clearly, they believe speciation to be a relatively infrequent event in the genealogical network. But splits, which are the speciation events that they envisage, are much more frequent than they seem to assume. As we have shown, temporary splits are so abundant that, if they were taken seriously as speciation events, they would give species only fleeting life spans. But even if we restrict speciation events to permanent splits, the life spans of the resulting species would be too short to meet our intuitions. After all, the extinction of any isolated branch of the genealogical network, no matter how small its membership, retrospectively renders a split in the network permanent (Figure 5.3). If we accepted every permanent split as a speciation event, humankind would be fragmented into two further species by every road accident in which a couple and all of its children perish. In many realistic scenarios, the life span of an internodon is shorter even than a generation. Far from needing Hennig's warning that species are not absolutely permanent, we require reassurance that they endure to any appreciable degree.

This constitutes a fundamental shortcoming of interpreting the internodon as species: the concept does not approximate closely to our intuitions about the life span of species. Together with the scarce degree of recognizability of members of internodons, this shortcoming weighs against identifying species with internodons.

Instead, we envisage a different role for internodons in the definition of a satisfactory species concept. We will show how a more inclusive and longerlived supra-organismal entity can be defined by reference to internodons. We will identify this entity, which preserves the properties of historicity and mutual exclusivity, as species. The definition of this more inclusive entity will, unlike that of the internodon itself, refer to criteria external to the structure of the genealogical network. These criteria can be of various kinds. In this paper, we have chosen to explore the possibility of using a morphological criterion for composite species in order to meet the practical need for species recognizability.

\subsection{A GENERALIZED WAY TO BUILD SPECIES FROM INTERNODONS}

The practice of defining species in terms of supra-organismal entities of some kind, rather than directly of organisms, is well established. The supraorganismal entity that is most frequently chosen for this task is the population. Species are defined as "composed of natural populations" by Mayr (1957: 13), as "systems of populations" by Dobzhansky (1970: 357), as "lineages, being ancestral-descendant sequences of populations" by Simpson (1961: 153) and Wiley (1981: 25), as "populations or groups of populations" by Rosen (1979: 277 ), and as "the smallest aggregation of populations (sexual) or lineages 
(asexual)" by Nixon and Wheeler (1990: 218). Similarly, de Queiroz and Donoghue (1988: 326) consider the basal units to be populations rather than species. Indeed, Nelson and Platnick have noted that "almost all definitions of the word 'species' that have been proposed utilize the word 'population'; species are populations, or groups of populations, that meet one or more criteria." (Nelson and Platnick, 1981: 11).

Nelson and Platnick continue with a warning, however: "But the word 'population' is itself in need of definition, and is fully as difficult to define as the word 'species'." (ibid.). Clearly, a definition of species based on a less inclusive supra-organismal entity is worthless if the latter entity is not itself precisely defined. But we have at our disposal rigorously defined supraorganismal entities, which moreover are mutually exclusive and historically continuous: the internodons. There is no other well-defined supra-organismal entity on offer in the literature, let alone one having the characteristics of mutual exclusivity and historicity. Henceforth in this paper, the concept of the internodon will be not a tentative model of a species, but rather a building block out of several of which a species will be composed.

A diagram of the internodons that have resulted from a sequence of permanent splitting events shows a phylogenetic tree of internodons. (It is legitimate to use the term "phylogenetic tree" for both the mapping of the relations among internodons and that of the relations among species, since these do not form networks, unlike relations among organisms.) Figure 5.4a depicts such a tree, and should be interpreted as representing the frequent permanent splits in the genealogical network and the internodons' consequent short life spans.

We conceive of a species as the set of the organisms belonging to several consecutive internodons in the phylogenetic succession, identified and grouped together by some procedure. We will call the species yielded by this concept composite species. Composite species originate with the coming into being of particular internodons: we will call each of these internodons the originator internodon of its species.

Let us stipulate that the originator internodons of species are identified by a particular quality $Q$ that they possess. Each originator internodon is allocated to a species together with all internodons that are its descendants and that do not exhibit $Q$. Every later internodon in that internodon lineage that exhibits quality $Q$ is the first internodon of a fresh species.

Quality $Q$, by which originator internodons are picked out from within the succession of all internodons, could be taken to consist of any one of several different properties. It may relate to the fixation of a new character state in the internodon, to the organisms' loss of the ability to interbreed with members of other internodons, or to some other event. If composite species are to be diagnosable in practice in the genealogical network however, it will be most useful to identify $Q$ with a morphological property shown by the organisms 
that are members of originator internodons. This option will be developed in the next section.

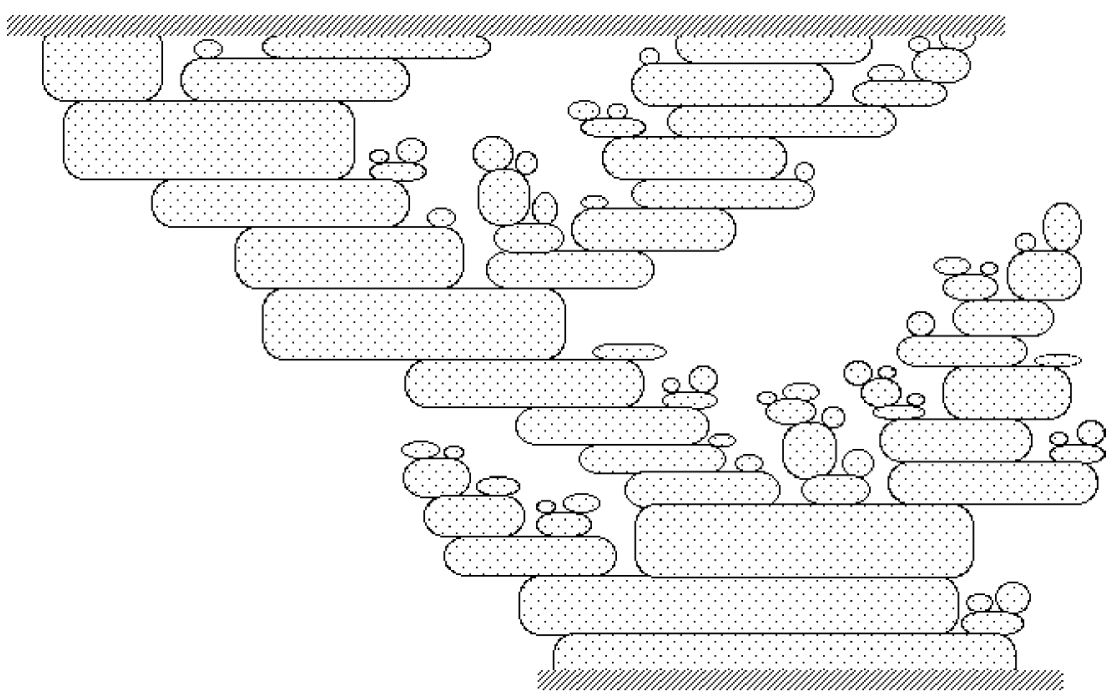

Figure 5.4a. A phylogenetic tree of internodons.

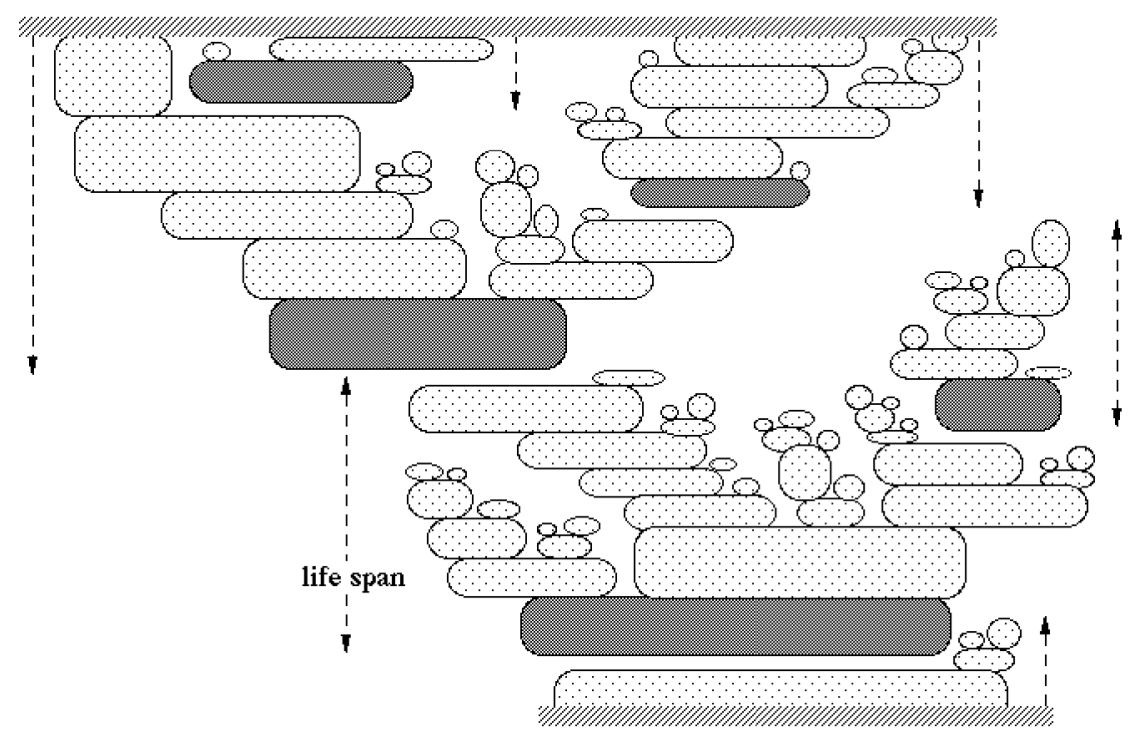

Figure 5.4b. Partitioning the phylogenetic tree into composite species. An originator internodon (shaded) is determined by some quality $Q$ of that internodon. A composite species survives until the extinction of the last of its internodons. 
Figure $5.4 \mathrm{~b}$, in which originator internodons (picked out arbitrarily at this stage) are shaded, shows how a phylogenetic tree of internodons is divided up into composite species. The life span of a composite species thus opens with the rise of its originator internodon. It extends over the life spans of several internodons in a sequence of descendant internodons. Finally, the life span of a composite species comes to an end with the extinction of the latest internodon that satisfies the following criteria: (a) it is a descendant of the originator internodon of the species, (b) it does not possess quality $Q$, and (c) there has appeared no other originator internodon in the branch of the phylogenetic tree of internodons between it and the originator internodon of the species. Of course, some internodons belonging to a species will become extinct before the life span of the species as a whole has come to an end. Nonetheless, the life span of the species as a whole does not end until all the organisms of every one of its internodons have died.

This means that the composite species will endure over, typically, several permanent splits in the genealogical network. Every permanent split brings about the ending of one internodon and the inauguration of at least two new ones, but not necessarily the inauguration of a new composite species: on the composite species concept, in other words, not every permanent split in the genealogical network is deemed a speciation event. For a permanent split to constitute a speciation event, at least one of the internodons that arise as a result of the split must have a particular property, identified arbitrarily at this stage as $Q$.

Consider, for instance, a group of organisms that becomes geographically detached from the main body of an interbreeding community, perhaps because it has colonized an island, and suppose that, as a matter of fact, a permanent split becomes established between the isolated group and the main community. On the composite species concept, successive internodons that arise within the group on the island are still conspecific with internodons of the main body, and remain so until one of them arises that has quality $Q$.

We have claimed that composite species retain both the property of historicity and that of mutual exclusivity shown by internodons. Let us see how these properties are transmitted to composite species.

A species delimited in the way we have described will have a historical beginning that coincides with the inception of its first internodon, and will possess historical cohesion owing to the continuity of the ancestral lines of internodons that originate in that first internodon. By virtue of this, the species will be a historical entity. In this respect, composite species do not differ from higher taxa. Each is composed of entities (internodons and species respectively) connected solely by ancestor-descendant relations, of which the members by definition do not interbreed and which therefore lack cohesion. (For discussion see Ereshefsky, 1991, and the references therein.) 
Composite species are clearly mutually exclusive, since internodons are themselves mutually exclusive, and we allocate each internodon to only one composite species. An alternative way of establishing that composite species are mutually exclusive makes use of the concepts of monophyly and paraphyly. The objection of Nixon and Wheeler (1990: 214), among others, that the concepts of monophyly and paraphyly should not be applied to entities below the species level, because such entities form reticulate groups, does not hold for internodons, since the member-organisms of different internodons do not interbreed.

The question whether species are monophyletic (posed by de Queiroz and Donoghue, 1988: 319) makes sense only in a more specific form, as the question whether species are monophyletic groups of some specified entities. For example, we may inquire whether a species is a monophyletic group of species (consisting of just one species), of organisms (as suggested by de Queiroz and Donoghue, 1988), or of internodons. The answer will differ with the entity chosen. A composite species that has no descendant species is the smallest possible monophyletic group of species, containing just one species. Simultaneously, every composite species could (if one is willing to disregard the objection of Nixon and Wheeler mentioned above) be seen as a polyphyletic group of organisms, except if its originator internodon has arisen with a single organism.

Here, however, we are interested in considering composite species as groups of internodons. A composite species is a paraphyletic group of internodons, except if it becomes extinct without leaving a descendant species, in which case it is a monophyletic group of internodons (Figure 5.5a).

Composite species are mutually exclusive only by virtue of the fact that every ancestral composite species (i.e. every composite species that has at least one descendant species) is a paraphyletic rather than a monophyletic group of internodons. Composite species would not be mutually exclusive if they were defined in every case to be monophyletic groups of internodons, since then there would be smaller (more recent) composite species wholly included within larger (longer-established) ones (Figure 5.5b). This is a general reason why, although being a monophyletic group of species is a desirable property of all higher taxa, which are intended to form hierarchies (Figure 5.5c), it is preferable to consider species as paraphyletic groups of internodons, since species are intended to be mutually exclusive in the genealogical network.

Once the genealogical network has been divided up into internodons, the only operation that is required to unite a number of internodons into a species is to identify the internodons that are the originator internodons of each composite species: in other words, to define quality $Q$. 

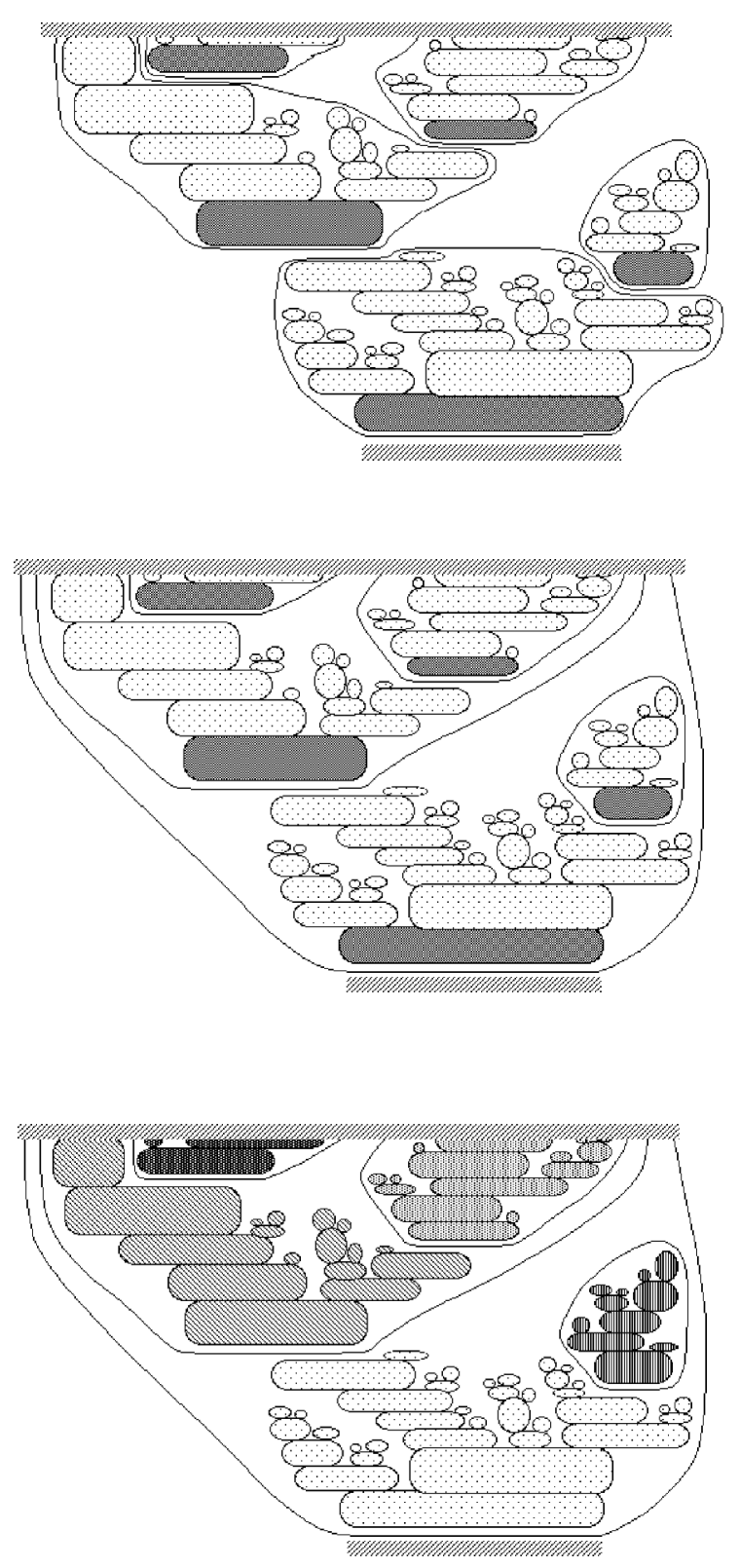

Figure 5.5a. Composite species, each arising with an originator internodon, are mutually exclusive. A composite species is a paraphyletic group of internodons, unless it becomes extinct without giving rise to a descendant species, in which case it is a monophyletic group of internodons.

Figure 5.5b. If all species were monophyletic groups of internodons arising with originator internodons, they would not be mutually exclusive: ancestral species would include successor species.

Figure 5.5c. Monophyletic groups of composite species include one another. This feature makes such groups suitable for hierarchical classification. The smallest monophyletic group of species consists of a single species. 


\subsection{A MORPHOLOGICAL DEFINITION OF QUALITY Q}

To summarize: we posit that the originator internodon of a species is distinguished by a particular quality $Q$, and is allocated to a species together with all internodons that are its descendants, up to but not including the next internodons that exhibit $Q$. Every later internodon in that internodon lineage that exhibits quality $Q$ is the first internodon of a fresh species.

The question remains, of course, what kind of quality $Q$ would best serve the purpose of uniting internodons into composite species?

One possibility is to seek to define $Q$ in terms of the logical apparatus developed by Kornet (1993). In that treatment, Kornet defines internodons (considered there as candidates for the status of species) by reference to the primitive terms of parenthood and chronological order of birth of organisms. If we took this route, $Q$ too would ultimately be reduced to these same primitive terms. But this option cannot, for logical reasons, be successful: Kornet's logical apparatus deliberately regards internodons as equivalent to one another, while the purpose of quality $Q$ is that it should uniquely identify certain internodons as being the first of their species.

What we need, therefore, is a defining criterion that is external to the logical apparatus used to partition the genealogical network into internodons. This means that we will construct a species concept defined jointly by two criteria: one (developed by Kornet, 1993) to group organisms into internodons, and one (under development in this paper) to unite internodons into composite species. Some previous species concepts have been flawed by their applying joint defining criteria that were incompatible, i.e. that did not always jointly apply, yielding indeterminate species boundaries (for discussion, see Kornet, 1993). However, our application of joint criteria does not introduce such flaws into our species concept. Quality $Q$ will be applied as a criterion only after the internodons have been delimited in the genealogical network, and will therefore be only a second-stage criterion.

Quality $Q$, marking originator internodons of composite species, can be defined in terms of biological concepts such as morphological characteristics or interbreeding ability. Because we strive to construct a species concept with maximal practical value, the most attractive option is to define $Q$ in terms of morphological criteria. After all, such criteria will make it possible to recognize composite species by familiar taxonomic methods. Here, we explore the possibility of defining a morphologically based quality $Q$ by reference to the fixation of character states.

Different authors describe fixation of a character state as taking place in different supra-organismal entities. For instance, de Queiroz and Donoghue (1990: 70-71) envisage fixation as occurring in a population, while Nixon and Wheeler (1990: 217) see it occurring in "terminal lineages" and "clades". 
Clearly, in our model, the supra-organismal entity in which fixation occurs is the internodon.

We now define quality $Q$ as the property that an internodon has by virtue of the fact that a character state becomes fixed within it. On this definition, a composite species originates with an internodon in which the fixation of a character state occurs, and endures (barring its extinction) until and including the internodon before the next internodon in which the fixation of a character state occurs (that is, the next internodon that also shows quality $Q$ ).

\subsection{CHARACTER STATES AND FIXATIONS}

For completeness, we must specify which "character states" and "fixations" we accept for the purposes of defining quality $Q$. In this section, we provide a new and more rigorous account of these key taxonomic terms.

In our view, character states are to be understood as (single or multiple) genetic properties that find a phenotypic expression. (Genetic properties that have no observable expression are of little use in practical taxonomy and are therefore ignored in the present approach.) We will call the phenotypic expression of some set of genetic properties corresponding to a character state the manifestation of that character state. The manifestation of a character state is therefore an attribute that an organism shows by virtue of possessing that character state. Our terminology will assume that every character state can be recognized as a state of a particular character. In the symbolism that we will use, $A_{1}$ and $A_{2}$ are two states of the same character $A$, and have manifestations $a_{1}$ and $a_{2}$ respectively.

The finer definition of phenotypic expression depends on certain issues that we here leave open, namely what counts as a "morphological" and "observable" attribute. The notion of morphological attributes may include only macromorphological properties (such as having red petals) or also other detectable properties. If every detectable property is deemed to count as phenotypic expression, the red petals of two organisms may count as different phenotypic expressions if the chemical pathways resulting in their red petals are different.

How does a new character state come into existence? Consider two organisms $x$ and $y$, each possessing character state $A_{1}$ and showing its manifestation $a_{1}$, which produce an offspring $z$. Suppose that the genetic material of $z$ was affected by a mutation event, as a result of which $z$ possesses genetic properties different from those of its parents in virtue of which the latter possessed character state $A_{1}$. If these genetic properties of $z$ have a phenotypic expression that makes $z$ observably different from its parents, we 
say that a character state $A_{2}$ has originated in $z$ that in $z$ has the manifestation $a_{2}$.

A character state $A_{2}$, which originated with organism $z$, endures as long as there are descendants of $z$ alive that inherit it. It vanishes from the genealogical network when either there are no further descendants of $z$, or the character state $A_{2}$ has been replaced in all the extant descendants of $z$ by one or more further states of the same character $\left(A_{3}, A_{4}\right.$, etc.).

The identity of a character state is tied to its origin. If a set of genetic properties, which amounts to a character state and is already present in the genealogical network, originates afresh in the network by a separate mutation event, then what originates in this second event amounts to a new character state. In other words, a character state of one organism is non-identical to a character state of another organism if and only if there is no single ancestor organism from which they both inherited the state.

Two non-identical character states can have indistinguishable manifestations. For instance, the manifestation of a character state $A_{3}$ can be indistinguishable from those of $A_{1}$ or $B_{3}$; i.e. it may be that $a_{3}=a_{1}$, or $a_{3}=b_{3}$. (We interpret these phenomena as reversal and convergence respectively.) However, a new character state cannot, in the light of its definition, have a manifestation indistinguishable from that of its immediate ancestor: a new character state is said to originate only in virtue of the fact that its manifestation differs from that of its immediate ancestor.

We have chosen the fixation of a character state as the criterion for $Q$ for its value for practical taxonomy. In order to obtain maximal recognizability for composite species, it is useful to distinguish among three senses in which a character state could be said to have become fixed. We shall call these full fixation, near fixation, and majority fixation. We will now define these, and examine which form of fixation best allows us to identify originator internodons on morphological criteria.

Of course, not every character state that arises will become fixed. Where necessary, we will distinguish a character state that becomes fixed by an asterisk (e.g., $\left.A_{3}{ }^{*}\right)$.

The best way of judging how well a form of fixation delivers diagnosability of originator internodons is by asking to what extent the period of fixation of a character state $-A_{5}{ }^{*}$, say-overlaps with the interval in which $A_{5}{ }^{*}$ has the highest frequency in the historical succession of states of character $A$. The period of fixation of a character state $A_{5}{ }^{*}$, for any form of fixation, is the time interval between the fixation of $A_{5}{ }^{*}$ and the fixation of the next state of character $A$ that happens to become fixed. 
The full fixation of a character state $A_{5} *$ in an internodon is the completion of the replacement of the previous state $A_{1}^{*}$ to have become fixed (Figure 5.6), or of several previous states of character $A$ that may have been present together, by $A_{5}{ }^{*}$ in every member organism of the internodon living at a certain time. Note that, while the replacement of some character state $A_{1}{ }^{*}$ by $A_{5}{ }^{*}$ is completed within some particular internodon, its frequency may well have been building up gradually in a succession of internodons; i.e. character state $A_{5}{ }^{*}$ may well have originated in an organism belonging to a relatively distant ancestral internodon.

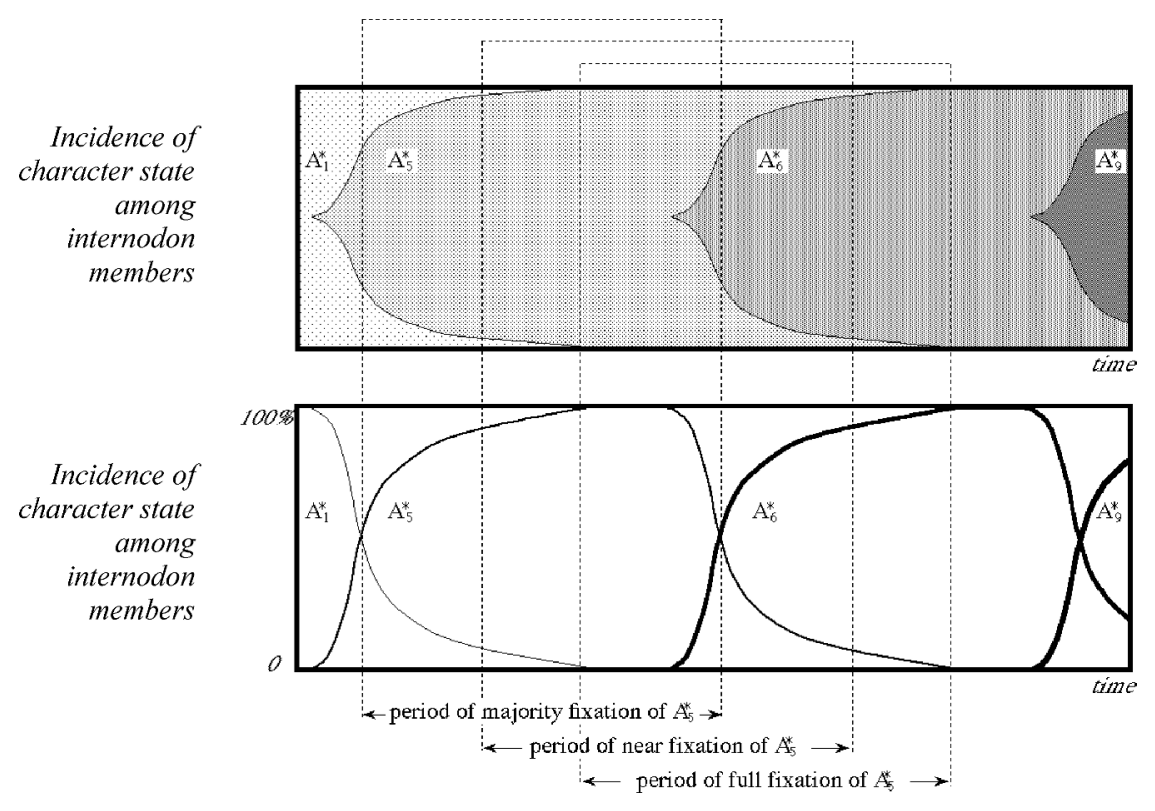

Figure 5.6. The incidence of each character state in a succession of internodons rises and falls in time. The periods of fixation associated with the three forms of fixation discussed in the text correspond to different intervals in this process.

The period of full fixation of a character state $A_{5}{ }^{*}$ extends from the time at which character state $A_{5}{ }^{*}$ first reaches an incidence of $100 \%$ among the thenliving members of an internodon to the time at which a later state of character $A$ reaches $100 \%$ incidence, typically in a successor internodon (Figure 5.6). A comparison of the period of full fixation of $A_{5}{ }^{*}$ with the time during which $A_{5}{ }^{*}$ achieves its highest frequencies among members of an internodon reveals that full fixation is an unsuitable form of fixation to which 
to tie property $Q$. The full fixation of a character state $A_{5}{ }^{*}$ typically occurs late in the career of the character state, after it has been present for long periods in a large proportion of the members of successive internodons. This is because the character state $A_{1}{ }^{*}$, the latest predecessor character state of $A_{5}{ }^{*}$ that became fixed, will typically persist among members of successive internodons at low frequencies well after its heyday. This means that, before the internodon in which $A_{5}{ }^{*}$ achieves full fixation, there may have been many internodons of which the organisms were already characterized by $A_{5}{ }^{*}$.

To remedy this shortcoming, we could relax the demand that, in order to be deemed to have reached fixation, a character state should attain 100\% incidence, and be content with a specified lower incidence. A character state's first reaching this specified incidence would constitute its near fixation (Figure 5.6). For character state $A_{5} *$ to achieve near fixation, it is therefore not necessary for the previous character state to have become fixed, $A_{1}{ }^{*}$, to disappear entirely. Near fixation still has two disadvantages for our purposes, however. The period of near fixation of $A_{5}{ }^{*}$ corresponds to the interval during which $A_{5}{ }^{*}$ achieves its greatest frequency more closely than does its period of full fixation, but it still leaves out many organisms with character state $A_{5}{ }^{*}$. In addition, stipulating a precise frequency at which the near fixation of $A_{5}{ }^{*}$ occurs would be arbitrary.

We therefore turn to the third option, majority fixation. We define the majority fixation of character state $A_{5}{ }^{*}$ as the event in which $A_{5}{ }^{*}$ for the first time in a single succession of internodons in the network reaches relative majority, i.e. a frequency greater than that of any other state of the same character then represented among members of the internodon. As can be seen from Figure 5.6, the period of majority fixation of character state $A_{5}{ }^{*}$ coincides more closely with the interval in which the frequency of $A_{5}{ }^{*}$ attains its highest values. This means that the originator internodon of the species which is to be characterized by character state $A_{5}{ }^{*}$ will be picked out more easily in the succession of internodons: this is done by locating the internodon in which for the first time a majority of the organisms living at any one time shows $A_{5}{ }^{*}$. Of course, it would be easy to mistake character state $A_{5}{ }^{*}$ for some other character state, if this other character state had manifestations indistinguishable from those of $A_{5}{ }^{*}$; nonetheless, this other character state is non-identical to $A_{5}{ }^{*}$ if, as we explained earlier, it had an independent origin in the genealogical network.

If character state $A_{5}{ }^{*}$ achieves majority fixation in some internodon, it can happen that, after a dip in its frequency, $A_{5}{ }^{*}$ achieves relative majority incidence also in an internodon which is a descendant of the first one. However, this event does not constitute majority fixation, in view of our 
stipulation that the majority fixation of a character state occurs only when it achieves relative majority incidence for the first time in any succession of internodons in the genealogical network. Without this stipulation, a character state that achieved relative majority incidence on several occasions separated by periods of lower frequency would have to be judged to have become fixed, and therefore to have given rise to a new species, on all those occasions. It is undesirable to associate the origin of a new species with perhaps very small oscillations of a frequency around a boundary value. (These observations hold also for near fixation.)

Nonetheless, a character state can achieve fixation of any of our three kinds more than once in a genealogical network. For instance, a character state $A_{5}{ }^{*}$ that has at some time achieved majority fixation in an internodon can achieve majority fixation at a later time in an internodon that is not a descendant of the first one. If this happens, each internodon in which $A_{5}{ }^{*}$ achieves fixation is an originator internodon. We will call this phenomenon parafixation.

In Figure 5.6, the succession of character states is idealized in at least three ways. First, Figure 5.6 does not show the incidence of the constantly arising and declining states of the character that never attain frequencies high enough to permit fixation. These would be contained in a band at the foot of the diagrams. Second, the diagrams portray character states as becoming fixed in the same chronological order as that in which they originate and disappear. In reality, this correlation will not always hold: a state $A_{1}{ }^{*}$ may arise earlier than $A_{5}{ }^{*}$, but remain for longer at low frequencies, and therefore become fixed after $A_{5}{ }^{*}$, or outlive it. Third, Figure 5.6 shows periods of fixation of the same duration. None of these diagrammatic simplifications invalidates our conclusion that majority fixation is a good basis for a definition of quality $Q$.

Majority fixation enables us also to deal adequately with a phenomenon that will here be called scrolling, while full and near fixation do not. In scrolling, successive new states of a character arise frequently enough that, while a particular state is still far from full or near fixation, the next state that will eventually become fixed has already appeared in some organisms (Figure 5.7). In this scenario, full or near fixation might well never occur, and yet a succession of internodons could still witness a succession of distinguishing character states. Species arising with an internodon marked by full or near fixation will then come to include many organisms lacking the character state by which the species is characterized. Majority fixation treats this phenomenon differently: every one of the character states that reaches relative majority characterizes a composite species, so species generated during a period of scrolling will not lose their diagnosability. 


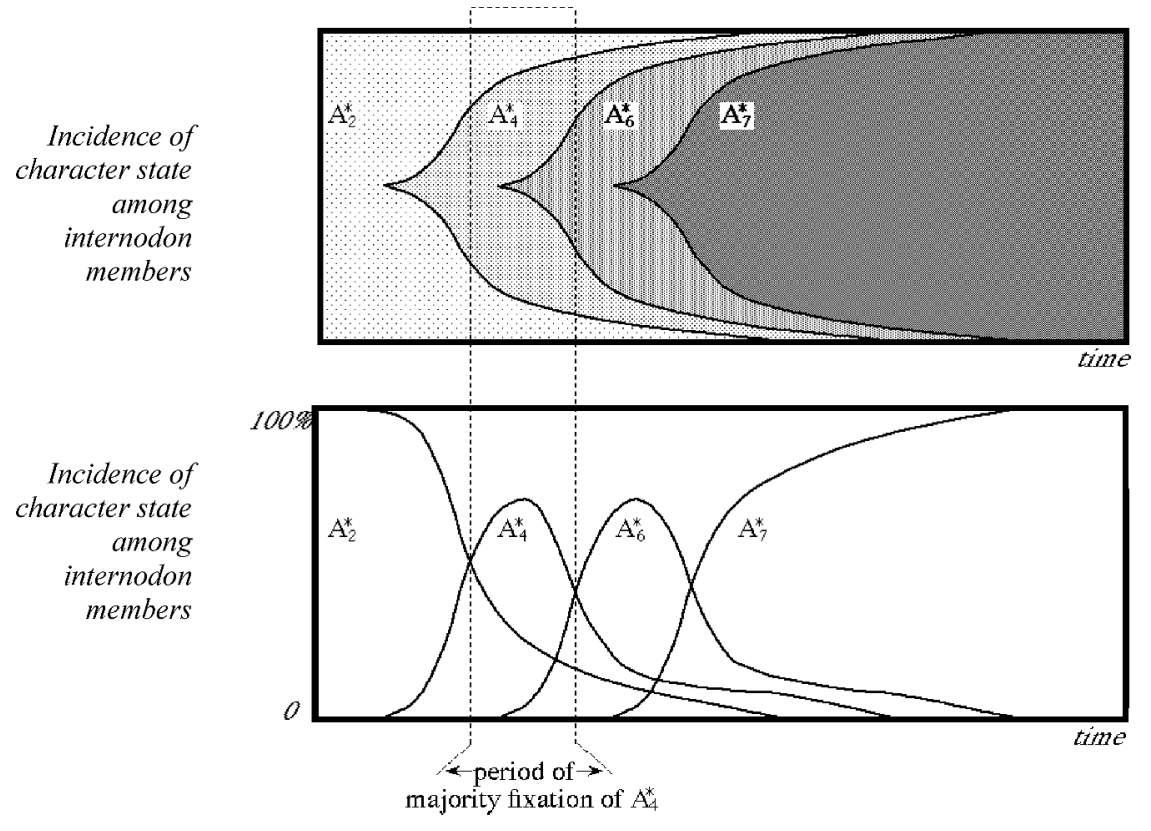

Figure 5.7. Majority fixation is the only form of fixation able to deal satisfactorily with the phenomenon of scrolling.

For practical applicability, therefore, we should indeed select as quality $Q$, which identifies originator internodons, the property of an internodon that a character state achieves majority fixation within it. Our assessment that majority fixation has the virtues described above does not depend on our endorsing any particular model of the evolution of novel character states, in the range stretching from gradualism to punctuated equilibria theory. Majority fixation offers good diagnosability in each of these cases. Whereas we opt for majority fixation on the strength of its diagnosability, we acknowledge that full fixation has a greater evolutionary importance than near and majority fixation, since it results in the disappearance of a previous character state.

The composite species concept stipulates that each composite species corresponds to one originator internodon. According to the composite species concept, therefore, each composite species in principle corresponds to the fixation of one character state. It may occur that more than one character state becomes fixed in a single originator internodon, though we expect this to be uncommon, in view of the short life span of internodons. In this case, we might speak of "double" speciation, resulting in "superposed" composite 
species. In practice, of course, we will deal with such superposed species as if they were single species, diagnosable by more than one character state.

\subsection{SUMMARY OF DEFINITIONS}

We are now able to state the definition of composite species:

A composite species is the set of all organisms belonging to an originator internodon, and all organisms belonging to any of its descendant internodons, excluding later originator internodons and their descendant internodons.

This definition refers to the notions of internodon and originator internodon. We define internodon as follows:

An internodon is a set of organisms such that, if it contains some $\operatorname{organism} x$, it contains all organisms that have the INT relation with $x$, and no other organisms.

Less formally, it may be conceived as a part of the genealogical network contained between two successive permanent splits, or between a permanent split and an extinction. (For further elucidation, see Kornet, 1993, and Kornet et al., 1995.)

Lastly, an originator internodon is an internodon distinguished by having some quality $Q$. In this paper, we interpret quality $Q$ as the property of an internodon that a character state achieves majority fixation in it. On this interpretation, an originator internodon is an internodon in which a character state achieves majority fixation.

In the remainder of this paper, we explore some of the implications of the notion of composite species, and investigate how it may be incorporated into extant phylogenetic practice.

\subsection{COMPOSITE SPECIES CONTRASTED WITH MORPHOLOGICAL SPECIES}

Whereas we use morphological criteria in the delimitation of composite species in the genealogical network, ours is emphatically not a morphological species concept. This section points out the differences between these two concepts of species. Thanks to these differences, the composite species concept avoids some of the problems that affect the morphological concept, such as its lack of sharp boundaries and its ahistoricity (Mayr, 1942: 115-118; Hull, 1976; see also Kornet, 1993).

Our criterion for species membership is morphological in the sense that, in applying it, regard must be paid to morphological attributes of organisms, since it is through examination of these attributes that one detects the fixation of a character state in an internodon. On the other hand, our criterion is not 
morphological if by this term one means that the species membership of an individual organism can be decided by looking at nothing but its morphological attributes. The function of our morphological criterion is to allocate organisms to species in virtue of the internodons to which they belong. The criterion has regard not primarily for the morphological attributes of individual organisms, but rather for the properties of an internodon as a whole, such as its property of being an internodon in which the fixation of a character state occurs.

The most convincing way of showing the difference between our species concept and the morphological concept is by noting that, in our concept, an organism's showing $a_{2}$, the manifestation of a character state $A_{2}$, is neither necessary nor sufficient for it to be allocated to the composite species associated with the fixation of $A_{2}$. By contrast, in any pure morphological concept, an organism belongs to the species defined in terms of one or more particular attributes if and only if it possesses those attributes. We shall now demonstrate that showing a particular manifestation is neither necessary nor sufficient for an organism to be allocated to a composite species.

There are two reasons why the possession of manifestation $a_{2}$ of character state $A_{2}$ is not necessary for an organism to be a member of the composite species defined by the fixation of $A_{2}$.

First, it is unlikely that a character state should become fixed in the first generation of an originator internodon. Because of this, even if full fixation of $A_{2}$ occurs in this internodon, some of the earliest-born members of that internodon are likely to lack the character state by the fixation of which the species is identified. (For discussion of a similar phenomenon in phylogenetic species, sometimes called a "paradox", see Nelson, 1989: 286, and de Queiroz and Donoghue, 1990: 68-69.) These organisms will not show the manifestations given by character state $A_{2}$.

Second, even after character state $A_{2}$ has become fixed within an internodon, if the form of fixation to which quality $Q$ is tied is either majority or near fixation, $A_{2}$ need not be possessed by, and therefore its manifestation $a_{2}$ need not be shown by, $100 \%$ of the member organisms of the internodon.

These are the reasons why it is not necessary for a member organism of the composite species associated with some character state $A_{2}$ to show the manifestations given by $A_{2}$. Now let us turn to consider whether an organism's showing manifestations indistinguishable from those given by $A_{2}$ is sufficient to compel its allocation to the composite species associated with $A_{2}$. There are two reasons why it is not.

First, a character state $A_{2}$, which becomes fixed in a particular internodon, can have spread also to branches of the genealogical network different from 
that on which this internodon is located. If it has, organisms outside the species associated with the fixation of $A_{2}$ can possess $A_{2}$ and therefore show the manifestations given by $A_{2}$.

Second, branches of the genealogical network other than the one in which $A_{2}$ has become fixed can contain organisms possessing character states that are non-identical to $A_{2}$, but that give organisms manifestations indistinguishable from those given by $A_{2}$. In this case, organisms outside the species associated with the fixation of $A_{2}$ can show the manifestations typical of $A_{2}$, despite not actually possessing $A_{2}$. This phenomenon, which we call convergence, will be discussed further in the next section.

This implies that an organism's possession of manifestations indistinguishable from those given by character state $A_{2}$ is not sufficient to allocate it to the composite species associated with the fixation of $A_{2}$, even if the organism is contemporaneous with the internodon in which the fixation takes place.

\subsection{APPLICATION OF THE COMPOSITE SPECIES CONCEPT IN CLADISTIC PRACTICE}

In this section, we aim to show that the composite species concept is compatible with actual cladistic practice, and that furthermore this species concept provides deep justifications of assumptions and procedures used in phylogeny reconstruction. To do this, we set up a hypothesized phylogenetic tree of internodons and investigate to what extent the taxa identified by cladistic practice correspond to composite species.

Figure 5.8a gives the phylogenetic tree of internodons that we will use for the test. This tree contains several composite species. The originator internodon of each species is one in which, as we envisage in our definition of $Q$, a state of a character becomes fixed. In the diagram, we deal with states of characters $A$ to $G$. Each state is denoted by a subscript numeral. The time at which one of these character states becomes fixed is marked in the diagram by its name, such as $A_{3}{ }^{*}$.

Each of the character states has a manifestation, represented by, e.g. $a_{3}$. As explained in Section 5.5, the manifestation of a character state is an attribute that an organism shows in virtue of possessing that character state.

Each species in Figure 5.8a is characterized by the manifestation of the character state that became fixed in its originator internodon. These morphological attributes of species are indicated in Figure 5.8a by the different shadings, distinguishing the several internodons of each composite species. These attributes are what, in practice, will be used to allocate a given organism 
to one of the composite species. Each of the character states $A_{3}{ }^{*}, B_{9}{ }^{*}, C_{8}{ }^{*}$, $D_{7}{ }^{*}$, and $E_{4}{ }^{*}$ gives, to the organisms that possess it, manifestations that are different from those given by the other character states.

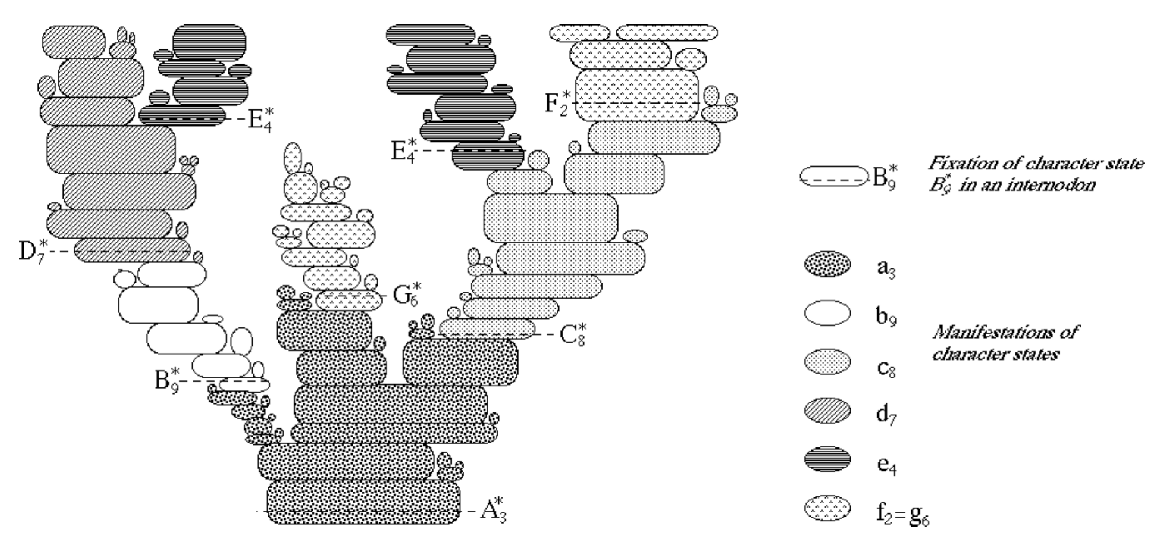

Figure 5.8a. A postulated phylogenetic tree of internodons. Internodons originate whenever a permanent split occurs in the genealogical network. In some of the internodons a character state has become fixed. The shading represents the manifestations of the character states. Character state $E_{4}{ }^{*}$ has become fixed twice. Character states $G_{6}{ }^{*}$ and $F_{2}{ }^{*}$ have similar manifestations.
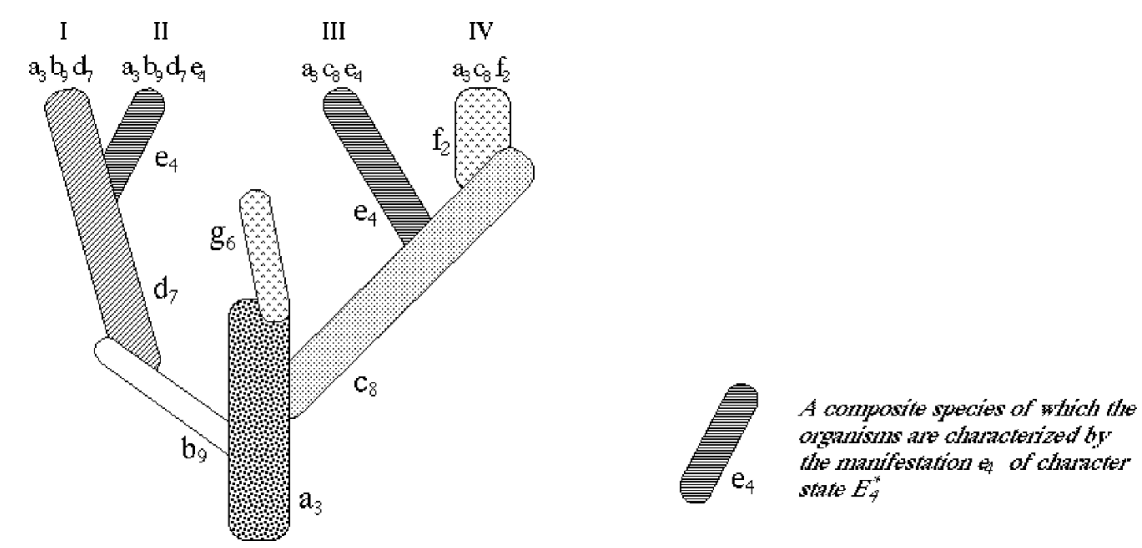

Figure 5.8b. A representation of the phylogenetic tree of species (the historical sequence of speciation events) drawn from the postulated phylogenetic tree of 
internodons of Figure 5.8a. Note that composite species speciate not by splitting up, but by branching off.

\begin{tabular}{l|llllll} 
& $\mathrm{a}_{3}$ & $\mathrm{~b}_{9}$ & $\mathrm{c}_{8}$ & $\mathrm{~d}_{7}$ & $\mathrm{e}_{4}$ & $\mathrm{f}_{2}$ \\
\hline OG & 0 & 0 & 0 & 0 & 0 & 0 \\
I & 1 & 1 & 0 & 1 & 0 & 0 \\
II & 1 & 1 & 0 & 1 & 1 & 0 \\
III & 1 & 0 & 1 & 0 & 1 & 0 \\
IV & 1 & 0 & 1 & 0 & 0 & 1
\end{tabular}

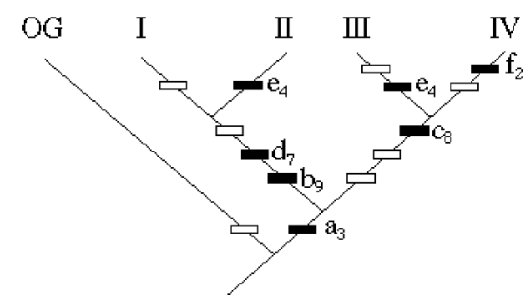

Figure 5.8c. Data matrix and cladogram obtained from the organisms of the recent time slice of the phylogenetic tree of species of Figure $5.8 \mathrm{~b}$.
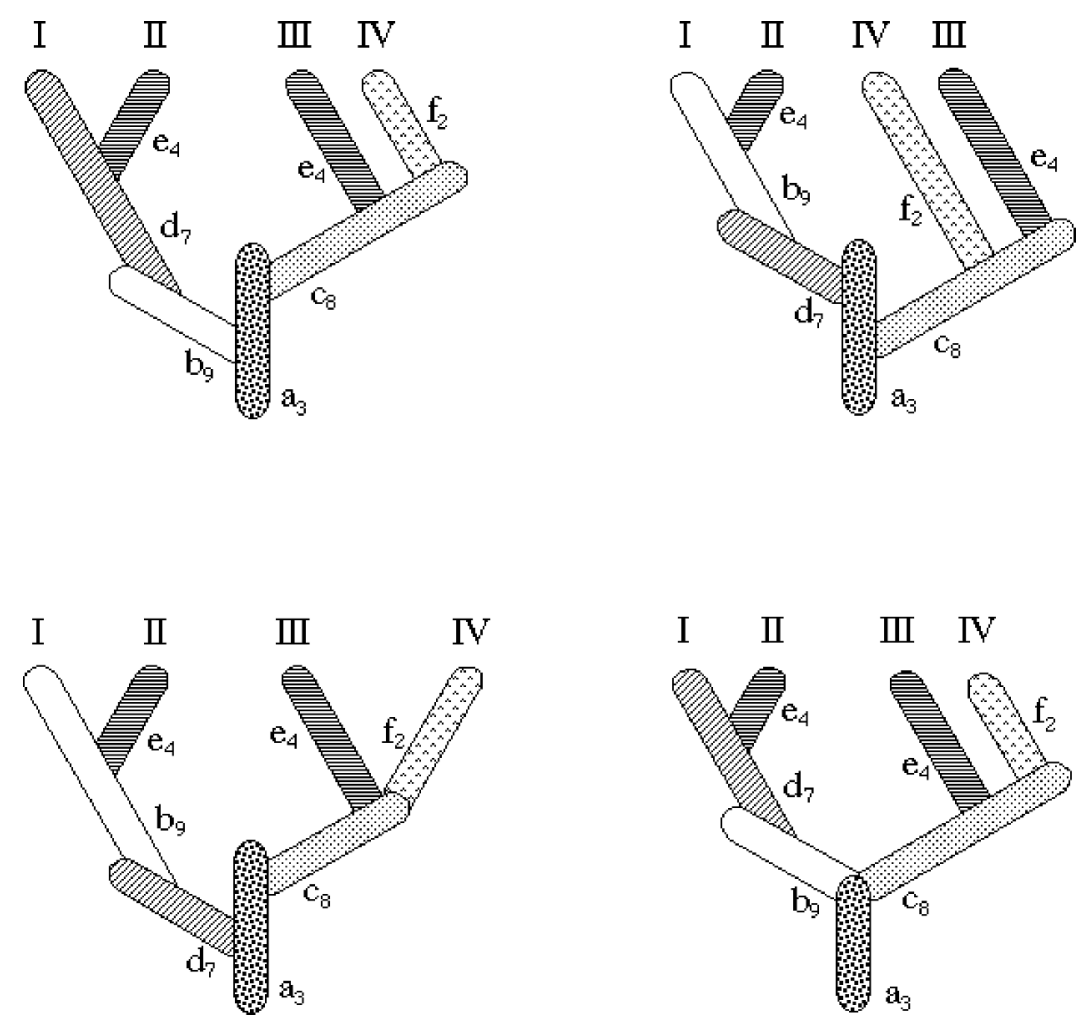

Figure 5.8d. Four reconstructions of the phylogenetic tree of composite species, out of the 27 that are compatible with the cladogram of Figure $5.8 \mathrm{c}$. 
Figure 5.8a shows two cases of homoplasy, or pairs of character states that, by the similarity of their manifestations, falsely suggest a common history of the taxa in which they are found. One case is due to the fact that, as our scenario envisages, $F_{2}{ }^{*}$ and $G_{6}{ }^{*}$ give to the organisms that possess them indistinguishable manifestations: $f_{2}=g_{6} . F_{2}{ }^{*}$ and $G_{6}{ }^{*}$ are different character states, in virtue of having had different origins in mutation events in the genealogical network. This case of homoplasy is due to convergence, i.e. to the rise of character states having similar manifestations but different ancestor character states (along the lines of Wiley, 1981: 12). The second case of homoplasy is due to the fact that character state $E_{4}{ }^{*}$ has become fixed twice, in two separate branches, qualifying two internodons as originator internodons and therefore giving rise to two composite species that are characterized by $E_{4}{ }^{*}$ and thus have the same manifestation. This is a case of homoplasy, since the presence of $E_{4}{ }^{*}$ on two different branches falsely suggests a common history of the taxa concerned. But it is not a recognized form of homoplasy: we propose to call this a case of parafixation. Parafixation is obtained when two organisms lying on separate branches of a phylogenetic tree show indistinguishable attributes in virtue of possessing one character state in common. For character state $E_{4}{ }^{*}$ to become fixed in two internodons, as illustrated in Figure 5.8a, the mutation event in which it originated must have occurred earlier in the phylogenetic tree: more precisely, it must have taken place in an internodon no later than the last internodon that is an ancestor of both the internodons in which $E_{4}{ }^{*}$ eventually becomes fixed. Clearly, parafixation is different from parallelism: while parallelism is the fixation of two character states with morphologically indistinguishable manifestations that developed from the same ancestor character state, parafixation is the fixation of one character state on two different branches.

Figure 5.8b depicts the phylogenetic tree of composite species that corresponds to the phylogenetic tree of internodons in Figure 5.8a. The attributes characteristic of each species shown in Figure 5.8a are shown here too, by the shadings as well as by the lower-case letters. Note that, as we shall discuss in the next section, composite species branch off and do not split up. Save for their latest time slice of reasonable thickness, the phylogenetic trees in Figures $5.8 \mathrm{a}$ and $5.8 \mathrm{~b}$ are not accessible to the taxonomist. The numerals I to IV identify the species that are extant now, at the time of the taxonomic investigation that we here envisage.

How is this model related to taxonomic practice? To diagnose extant composite species, the following procedure suggests itself. The taxonomist, examining the latest organisms belonging to species I to IV, compiles a record of their attributes against their locations. The first task is to diagnose internodons. Groups of organisms might most plausibly be supposed to 
constitute separate internodons if they are isolated from one another. Then, combinations of fixed character-state manifestations should be found such that every entity believed to be an internodon shows one such combination. All internodons whose members show the same combination of fixed characterstate manifestations are allocated to the same composite species. It should not be expected that every composite species has a fixed character-state manifestation that no other species has (i.e. that it has an autapomorphy of its own). For instance, in Figure 5.8b, species I, because it is ancestral, has a unique combination of fixed character-state manifestations $\left(a_{3} b_{9} d_{7}\right)$, but no character-state manifestation in this combination is unique to it.

The combinations of fixed character-state manifestations found for the extant composite species are recorded in a data matrix (Figure 5.8c). From the data matrix, by cladistic analysis, the taxonomist hypothesizes cladograms. (On cladograms, see e.g. Eldredge and Cracraft, 1980: 19-85.) In our model, a cladogram is a candidate reconstruction of the distribution of acquisitions of character states over the branches of the genealogical network; in other words, it is a map of the sequence of character-state fixations in branches of the genealogical network. Figure 5.8c shows the cladogram that cladistic analysis programs, such as PAUP (Swofford, 1991) and CAFCA (Zandee, 1991), indicate to be the best-supported solution admitted by the data matrix shown.

Various possible phylogenetic trees of species can be inferred from a cladogram. (Compare Cracraft, 1974; Nelson and Platnick, 1981: 169-183; and Wiley, 1981: 104-108.) Which particular trees are obtained depends on the concept of species used.

On our interpretation, the segments of a cladogram do not correspond to species, and the points at which these segments originate do not correspond to speciation events. (More about this at the end of the present section.) A segment with one character-state acquisition corresponds to a single speciation event. Empty segments of the cladogram, i.e. segments on which no characterstate acquisitions are marked (such as terminal segment I), should be interpreted as indicating that an ancestral species survived one or more speciation events in which daughter species originated. Finally, segments on which more than one character-state acquisition is marked correspond in principle to an equal number of speciation events, and therefore indicate in principle the existence of an equal number of composite species that are descendants of one another. If this is so, the segment labelled with characterstate manifestations $b_{9}$ and $d_{7}$ in Figure 5.8c represents a sequence of two species, one being the descendant of the other. The exception is constituted by the case of double speciation (see Section 5.5), in which more than one character state happens to become fixed in the same originator internodon, giving rise to superposed species. 
On the composite species concept, a single cladogram may be compatible with several phylogenetic trees, for two reasons. First, a cladogram admits several trees if it has segments on which more than one character-state acquisition appears, since it is impossible from the data contained in the latest time slice to ascertain when these character states were acquired. Because of this, we cannot reconstruct the order in which the composite species arose, and whether any of them were superposed species. Second, the cladogram cannot indicate the order in which different species possessing the same ancestor species branched off from that ancestor. However, neither homoplasies (convergences, reversals, parallelisms, or parafixations) nor the empty segments in a cladogram increase the number of phylogenetic trees that are compatible with a cladogram.

From the morphological data available for the latest time slice, it is not possible to determine which of the candidate trees describes the historical events. To discriminate further, we would require extra data: those that may be acquired from earlier time slices, such as by palaeontology. It will also be impossible on the basis of the data matrix to decide whether the fact that taxa II and III are characterized by (i.e. have as autapomorphy) indistinguishable manifestations ( $e_{4}$ in Figures 5.8c and 5.8d) is due to parafixation (as in fact our scenario stipulates in Figures $5.8 \mathrm{a}$ and $5.8 \mathrm{~b}$ ) or to convergence or parallelism. Since, however, we would consider taxa II and III two different species in either case, this uncertainty does not affect the construction of hypotheses of the phylogenetic tree of composite species.

By this procedure, 27 possible phylogenetic trees are obtained from the cladogram in Figure 5.8c (including nine trees in which the species characterized by $b_{9}$ and $d_{7}$ are "superposed": see Section 5.5). In Figure 5.8d, we show a sample of four of these trees. One of them, the first, reproduces the actual phylogenetic tree of species as we postulated it in Figures 5.8a and 5.8b, save for the species characterized by $G_{6}{ }^{*}$, which died out before the present, and of which therefore no trace survives in the cladistic data.

The superficial similarity of cladograms and phylogenetic trees may tempt some to interpret a cladogram as a stylized phylogenetic tree, in which each segment corresponds to a species, and in which each point at which a segment originates corresponds to a speciation event. This interpretation of a cladogram however, yields species (which we shall call cladospecies) quite different from composite species.

For example, if we interpreted each of the segments of the cladogram in Figure $5.8 \mathrm{c}$ as corresponding to a species, the resulting phylogenetic tree of cladospecies would be that illustrated in Figure 5.9a, and the postulated phylogenetic tree of internodons would correspondingly be partitioned as in Figure 5.9b. 

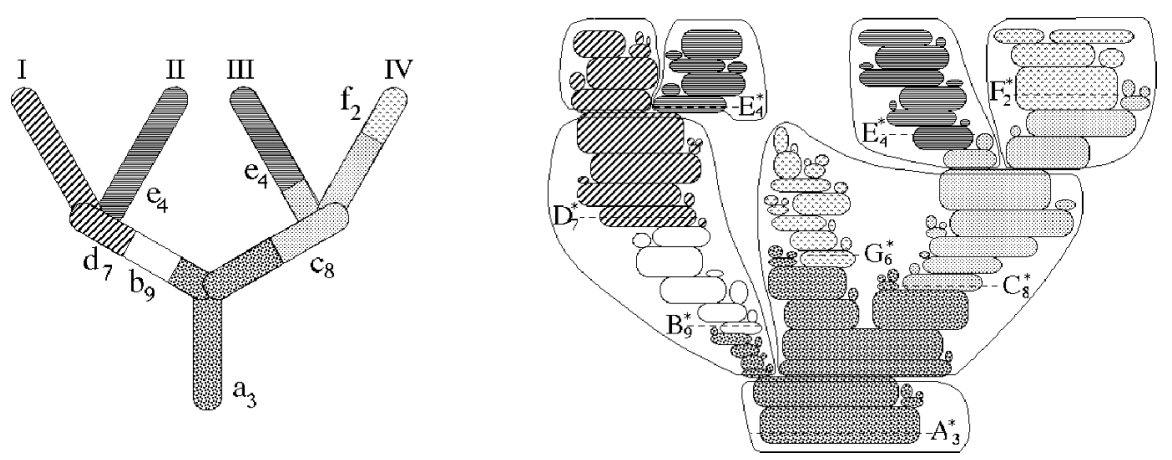

Figure 5.9. If segments in the cladogram of Figure $5.8 \mathrm{c}$ are interpreted as species, the phylogenetic tree of cladospecies is as shown here (left). This corresponds to a division of the phylogenetic tree of internodons into cladospecies (right). The speciation of cladospecies is necessarily dichotomous.

In the cladospecies, there is no guarantee that any character acquisitions that occur will be located in the first internodon, or even close to the beginning of species life spans. Where this does not happen, the diagnosability of these species will be lowered, as Figure 5.9b shows. Moreover, this interpretation of a cladogram condemns us to considering speciation as invariably dichotomous (as shown in the phylogenetic tree of cladospecies in Figure 5.9a), which is generally considered an artificial representation of speciation imposed by methodological principles. These are the reasons for which cladogram segments ought not to be construed as species.

\subsection{COMPOSITE SPECIES DO NOT SPLIT UP, BUT BRANCH OFF}

The Hennigian species concept requires the methodological principle, sometimes defended also as an empirical claim about speciation, that speciation should be seen as occurring by splitting up of branches (Hull, 1979: 425). In a splitting up, a new branch arises by the bifurcation of an extant branch of the genealogical network into two (or possibly more) new branches, which are siblings of one another. The rise of one successor branch is necessarily accompanied by the rise of a sibling of it, and the rise of these successor branches is necessarily accompanied by the ending of the ancestor branch. This is the way in which, by virtue of their definition, we envisage internodons to originate. However, if, as is generally accepted (Hull, 1979: 432), speciation may well occur through the isolation of a small interbreeding community, there is no justification for assuming that the ancestral species always becomes extinct in speciation (Figure 5.8b). 
On the composite species concept, speciation occurs by branching off: a successor branch can arise without the rise of any sibling branches of it, and without the ending of the ancestor branch. Any internodon (and therefore any internodon possessing $Q$ ) arises by the splitting up of a branch of the genealogical network into two. One of these branches is occupied by the internodon with quality $Q$, which is the originator internodon of a new composite species. What occurs to the internodon that is its sibling? There are two possible cases.

In by far the more common case, no character state becomes fixed in the sibling internodon. This internodon cannot be an originator internodon: rather, it must belong to the same species as the latest internodon that is the ancestor of both it and its sibling (see Figure 5.8a). Therefore, the ancestor species has survived the rise of the originator internodon of the new species. This means that the emergence of a daughter species does not imply the disappearance of its ancestor species: on the contrary, the ancestor species typically persists, at least for a while, after the speciation event, so that the life spans of an ancestor and daughter composite species overlap. It follows that composite species arise by branching off rather than by splitting up.

In by far the less common case, there occurs a fixation of a character state in the second internodon, as well as in its sibling. Here both the internodons are originator internodons, of different composite species; and each of the two originator internodons and the internodon from which they arose belongs to a different composite species. While this might be viewed as a splitting up, in fact every instance of splitting up is an instance of branching off: a particular branching off in which two branches arise simultaneously. In the light of this, we are warranted in both the cases described here to speak of speciation by branching off.

\subsection{THE COMPOSITE AND PHYLOGENETIC SPECIES CONCEPTS COMPARED}

The question may arise how the composite species concept is related to the phylogenetic species concept. The phylogenetic species concept is defined by Cracraft (1989: 34-35) as "an irreducible (basal) cluster of organisms, diagnosably distinct from other such clusters, and within which there is a parental pattern of ancestry and descent", by Nelson and Platnick (1981: 12) as "simply the smallest detected samples of self-perpetuating organisms that have unique sets of characters", and by Nixon and Wheeler (1990: 218) as "the smallest aggregation of populations (sexual) or lineages (asexual) diagnosable by a unique combination of character states in comparable individuals (semaphoronts)." 
The composite and phylogenetic species concepts show certain similarities, as follows.

First, both the composite and phylogenetic species concepts define species as collections of supra-organismal entities: these are internodons in the former concept, and populations, clusters, or samples of organisms in the latter. In both concepts, identifying a species in nature requires first that the component entities be picked out. As Nixon and Wheeler (1990: 218) state, "application of the phylogenetic species concept requires initial hypotheses of populations before relevant comparisons among individuals can be made", giving to populations the role that we give to internodons.

Second, both species concepts rely on similar diagnostic indicators to pick out these component entities in practice. Nixon and Wheeler (1990: 219) advise that populations can be "hypothesized initially on the basis of location and similarity of attributes". We likewise suggest (Section 5.8) that a group of organisms that share attributes and are isolated from other organisms should be hypothesized as constituting an internodon.

In both species concepts, component entities (internodons and populations, clusters, or samples) in the latest time slice are united into species on the basis of morphological similarity. We allocate all internodons that show the same combinations of fixed character-state manifestations to the same composite species. Likewise, Nixon and Wheeler (1990: 220) recommend that populations with the same set of character states be allocated to species, regardless of whether they interbreed.

In both species concepts, the morphological criterion used to group the component entities into species is the unique combination of character states (see Cracraft, 1983: 103; Nelson and Platnick, 1981: 12; Nixon and Wheeler, 1990: 218). A species "need not have even a single character that is unique to it", as Nelson and Platnick (1981: 12) put it and as we also allow.

Alongside these similarities, we see at least two important differences between the composite and phylogenetic species concepts.

The first is that the definitions of population, cluster, and sample of organisms used in the phylogenetic species concept are vague (see Section 5.3). It is therefore difficult to know where the boundaries of these entities lie in the genealogical network. A particular failing of these definitions is that they do not specify by what the life span of these entities is bounded. Because of this, the phylogenetic species concept meets difficulty in drawing boundaries in time between species in the genealogical network. How in the phylogenetic species concept does one demarcate an ancestor from a daughter species in the genealogical network? Because of its incapacity to answer this question, the phylogenetic species concept can be applied only to organisms in the latest time slice. In contrast, in the composite species concept the component entity of species receives a rigorous definition. The boundaries of 
both internodons and composite species in the genealogical network are therefore sharp, even in time.

The second important difference between the composite and phylogenetic species concepts is that, while in the composite species concept speciation is viewed as a branching off rather than a splitting up, the phylogenetic species concept is unclear on this point. In general, users of the phylogenetic species concept seem to regard it as interpreting speciation as dichotomous.

In the light of these similarities and differences, we consider the phylogenetic species concept as an approximation to the composite species concept that provides a less precisely defined theoretical framework and therefore less deep justifications of cladistic procedures and results. When it is applied to organisms in the most recent time slice, the approximation of the phylogenetic species concept to the composite species concept is quite close; but in the historical reconstruction of taxa by cladistic analysis, the approximation is loose.

\subsection{CONCLUSIONS}

Our attempt has been to construct a rigorously defined species concept that delivers species that are mutually exclusive, historical, and recognizable entities. To do so, we have taken a number of decisions for which there were alternatives. We have chosen, for instance, to identify quality $Q$ with a morphological quality, rather than with, say, interbreeding ability, and to associate $Q$ with the fixation of one character state, rather than more than one. We leave it to others to judge whether ours have been the optimal choices to reach our goal. We further discussed whether majority incidence or $100 \%$ incidence was the preferable notion to which to tie the fixation of a character state: while we have shown the merits of the former, we would not want the discussion of the composite species concept to be confined to this choice.

In conclusion, we draw attention to one peculiarity of the composite species concept. The origin of a composite species is tied to the achievement of fixation of a character state in an internodon. But a character state will achieve fixation much more frequently in small internodons than in large ones. Therefore new, small composite species will often arise with small internodons in which, by chance, a character state has high incidence. This effect is likely to manifest itself in the following two contexts.

First, if by a permanent splitting a small internodon arises of which the members happen to share a character state that is common locally but rare elsewhere, that internodon will be the originator internodon of a new, minuscule composite species. If this should happen frequently, "fringe species", defined by character states that are typically different from that 
defining the composite species from which they branch off, would arise continuously at the margins of interbreeding communities.

Second, if the incidence of a character state increases gradually in a branch of a genealogical network, that character state may achieve majority fixation earlier in smaller internodons than in larger ones. Then the coming into being of a larger composite species would be foreshadowed by the origination of many smaller "forerunner species" defined by the same character state. The composite species concept counts each of these as separate species, despite the fact that their member organisms are typically indistinguishable.

These features of the composite species concept might be removed by further extending the concept. One option is to stipulate that small internodons at the margins of a large species in which character states reach fixation in the ways described here do not originate new species, but are part of the species on the margins of which they develop. This stipulation could be achieved by formulating a third-stage criterion, based perhaps on interbreeding ability, to group these entities into species.

\section{ACKNOWLEDGEMENTS}

The first author presented the main conclusions of this paper at the Eleventh Meeting of the Willi Hennig Society, Paris, August 1992. We thank the participants for their constructive response. We also thank the editors of this volume and two referees for helpful suggestions. The investigations of the first author were supported by the Foundation for Biological Research $(\mathrm{BION})$, which is subsidized by the Netherlands Organization for Scientific Research (NWO).

\section{REFERENCES}

Cracraft, J. (1974). Phylogenetic models and classifications. Systematic Zoology 23: 71-90.

Cracraft, J. (1983). Species concepts and speciation analysis. Reprinted in Ereshefsky, 1992, pp. 93-120.

Cracraft, J. (1989). Speciation and Its Ontology: The Empirical Consequences of Alternative Species Concepts for Understanding Patterns and Processes of Differentiation. In: Otte, D. and J. A. Endler (Eds). Speciation and Its Consequences. Sinauer Associates, Sunderland, Mass. pp. 28-59.

de Queiroz, K. and M. J. Donoghue (1988). Phylogenetic systematics and the species problem. Cladistics 4: 317-338.

de Queiroz, K. and M. J. Donoghue (1990). Phylogenetic systematics or Nelson's version of cladistics? Cladistics 6: 61-75.

Dobzhansky, T. (1970). Genetics of the Evolutionary Process. Columbia University Press, New York. 
Eldredge, N. and J. Cracraft (1980). Phylogenetic Patterns and the Evolutionary Process: Method and Theory in Comparative Biology. Columbia University Press, New York.

Ereshefsky, M. (1991). Species, higher taxa, and the units of evolution. Reprinted in Ereshefsky, 1992, pp. 381-398.

Ereshefsky, M. (Ed.) (1992). The Units of Evolution: Essays on the Nature of Species. MIT Press, Cambridge, Mass.

Hennig, W. (1966). Phylogenetic Systematics. University of Illinois Press, Urbana.

Hull, D. L. (1976). Are species really individuals? Systematic Zoology 25: 174-191.

Hull, D. L. (1979). The limits of cladism. Systematic Zoology 28: 416-440.

Kornet, D. J. (1993). Permanent splits as speciation events: a formal reconstruction of the internodal species concept. Journal of Theoretical Biology 164: 407-435.

Kornet, D. J., J. A. J. Metz, and H. A. J. M. Schellinx (1995). Internodons as equivalence classes in genealogical networks: building-blocks for a rigorous species concept. Journal of Mathematical Biology 34: 110-122.

Mayr, E. (1942). Systematics and the Origin of Species. Dover, New York (reprinted 1964).

Mayr, E. (1957). Species Concepts and Definitions. In: Mayr, E. (Ed.). The Species Problem. American Association for the Advancement of Science, Washington, D.C. pp. 1-22.

Nelson, G. (1989). Cladistics and evolutionary models. Cladistics 5: 275-289.

Nelson, G. and N. I. Platnick (1981). Systematics and Biogeography. Columbia University Press, New York.

Nixon, K. C. and Q. D. Wheeler (1990). An amplification of the phylogenetic species concept. Cladistics 6: 211-223.

Ridley, M. (1989). The cladistic solution to the species problem. Biology and Philosophy 4: 1-16.

Rosen, D. E. (1979). Fishes from the uplands and intermontane basins of Guatemala: revisionary studies and comparative geography. Bulletin of the American Museum of Natural History 162: 267-376.

Simpson, G. G. (1961). Principles of Animal Taxonomy. Columbia University Press, New York (reprinted 1990).

Swofford, D. L. (1991). PAUP: Phylogenetic Analysis using Parsimony. Computer program distributed by the Illinois Natural History Survey, Champaign, Illinois.

Wiley, E. O. (1981). Phylogenetics: The Theory and Practice of Phylogenetic Systematics. John Wiley, New York.

Zandee, M. (1991). CAFCA, version 1.3c for Macintosh. User's manual distributed by the author. Institute of Theoretical Biology, Leiden.

\section{J. Kornet, Philosophy of the Life Sciences Group, University of Leiden}

James W. McAllister, Faculty of Philosophy, University of Leiden 